\title{
Sexual Dysfunctions in Depression
}

Shivananda Manohar J, TS Sathyanarayana Rao*, Suhas Chandran, Shreemit Maheshwari, Manju George, Suman S Rao and Fiaz Ahmed Sattar

Department of Psychiatry, JSS Medical College, JSS University, Mysore, India

\begin{abstract}
Sexual dysfunction is one of the common symptoms seen in depression. Presentations include decreased libido, erectile dysfunction, arousal difficulties and orgasm related dysfunctions. The probable causes of sexual dysfunction in such patients are complex and biological, psychological as well as psychosocial factors are likely to play an important role. Sexual dysfunction could also occur as an adverse effect of anti-depressants although it is difficult to establish whether the dysfunction is due to the illness or the medication. Awareness among professionals about sexual dysfunction is essential as it can have a profound impact in terms of relationship conflict, marital satisfaction, quality of life and compliance with treatment in patients with depression.
\end{abstract}

Keywords: Depression; Sexual dysfunction; Anti-depressant induced sexual dysfunction; Impact of sexual dysfunction

\section{Introduction}

Sexual behavior is a complex as well as an interesting interaction between biology and psychology. Together with eating and sleeping, sex is an important instinctual drive. Sexual life is an important factor in determining quality of life of a person. In a study done in US $94 \%$ of people opined sexual enjoyment added to quality of life in any age [1].

\section{Sexual Dysfunction in General Population}

The area of sexual dysfunction is under studied. Many factors influence prevalence of reported sexual dysfunction. First method of enquiry, in a study done on estimation of prevalence of sexual dysfunction, the estimated prevalence based on spontaneous reporting was $14 \%$, but the percentage of sexual dysfunction rose to $58 \%$ when directly enquired by the doctor [2]. Second the cultural background of the individual, which has an impact on gender roles, gender role expectations, explanations of sexual behaviour, explanatory models of sexual dysfunction and personal and cultural beliefs of healing. Third the perception of "normal" in terms of the individual perspective. Fourth being the knowledge about the availability of treatment options for sexual dysfunction [3].

A review done by Nathan on prevalence of sexual dysfunction in general population, found methodological problems in sexual surveys, he concluded only broad estimates can be done. In his review he reported prevalence of inhibited sexual desire was $16 \%$ for men, $35 \%$ for women; for erectile dysfunction and premature ejaculation the prevalence values were $10-20 \%$ and $35 \%$ of men, respectively; female orgasmic difficulties had a prevalence of 5-15\% [4]. A US study found that sexual dysfunction in the general population is more prevalent in women $(43 \%)$ than men (31\%). In this study, using latent class analysis, symptoms during the previous 12 months were grouped into three categories. In women, these were low sexual desire (22\% prevalence), arousal or excitement problems (14\%) and sexual pain (7\%); in men, they were premature ejaculation (21\%), erectile dysfunction (5\%) and low sexual desire (5\%) [5].

\section{Neurobiology of Sexual Response Cycle}

Human sexual response cycle is mediated by neurotransmitters and hormones. Neurotransmitters like serotonin, acetylcholine, nitric oxide and hormones like testosterone acting in specific brain structures like hypothalamus, limbic system and cortex, mediate sexual response cycle.

\section{Brain Structures Involved in Sexual Response Cycle}

Nucleus paragigantocellularis projecting directly into pelvic efferent neurons and interneurons in the lumbosacral spinal cord has been identified as important in orgasm, this area have also been implicated in SSRI induced anorgasmia in males and females. The raphe nuclei palidus,the magnus and parapyramidal region, and the locus ceruleus all project into lumbo sacral spinal cord. The medial Amygdala has been found to be associated with sexual behavior in females as well as sexual motivation in males. Studies have reported electrical stimulation of the Hippocampus resulting in erection while electrical stimulation of the septal region results in orgasm. Electroencephalographic studies have shown a right temporal activation when right handed persons were exposed to sexual stimuli [6].

\section{Hormones Involved in Sexual Response Cycle}

Plasma testosterone is necessary during desire phase in both men and women. Testosterone influences specific brain regions that are sensitive to internal and external clues. Blood levels of testosterone area affected by prolactin because testosterone release is directly inhibited by prolactin. Medications which increase prolactin (Tricyclic antidepressants, antipsychotics), will indirectly decrease testosterone. Hyper prolactinemia also increases GABAergic activity and endogenous opoids which affect libido and erection [7].

Cushing syndrome characterized by Hypercortisolism, is associated with depression, insomnia and decreased libido. Symptom severity is more when Hypercorisolism is associated with increased corticotrophin. Symptom severity is less when Hypercortisolism is associated with less corticotrophin.

Oxytocin facilitates penile erection when injected in specific areas like periventricular nucleus of hypothalamus, shortens ejaculation

*Corresponding author: Dr. TS Sathyanaryana Rao, Department of Psychiatry, JSS Medical College, JSS University, Mysore, India, Tel: +91 9845282399; E-mail: tssrao19@yahoo.com

Received: September 22, 2017; Accepted: September 27, 2017; Published: September 30, 2017

Citation: Manohar SJ, Sathyanarayana Rao TS, Chandra S, Maheshwari S George M, et al. (2017) Sexual Dysfunctions in Depression. Clin Depress 3: 125.

Copyright: (C) 2017 Manohar SJ, et al. This is an open-access article distributed under the terms of the Creative Commons Attribution License, which permits unrestricted use, distribution, and reproduction in any medium, provided the original author and source are credited. 
latency and post ejaculatory interval when injected centrally. In females either injected centrally or peripherally increases sexual behavior as measured by increasing lordosis [8]

Phremones are secreted from mouth, breast, anal outlet and urinary outlet in non-human mammals-it is secreted by vomeronasal structure, though vomeronasal structures have been demonstrated in humans but specific functions has not been found. Phremones acts as agents in terms of attracting, mating and identifying their sexual partners.

\section{Neurotransmitters Involved in Sexual Response Cycle}

Serotonergic system largely plays inhibitory role in all phases of sexual response cycle [9-12]. Serotonin's effects in the central nervous system are determined by the specific receptors activated.5hydroxytryptamine (5HT)-type2 and 5-HT3 receptors inhibit sexual activity while 5HT1 receptors stimulate sexual activity. Dopamine plays significant role in sexual response cycle, through its involvement in mesolimbic pathway and reward system. Activation of Nucleus accumbens and medial preoptic hypothalamic region by dopamine is essential for sexual motivation. Activation of paraventricular nucleus of hypothalamus by dopamine is essential for penile erection (Figure 1).

\section{Sexual Response Cycle in Depression}

Major depressive disorder negatively affects sexual response cycle. Around $40 \%$ of men suffering from major depressive disorder have reduced sexual interest and $20 \%$ of men have inability to reach orgasm or ejaculation. Functional MRI studies of depressed woman. Functional MRI (fMRI) of depressed women actively visualizing sexually stimulating imagery has shown less activation in certain brain regions (e.g., middle occipital gyrus, middle temporal gyrus, inferior frontal gyrus, insula, hypothalamus, septal area, anterior cingulate gyrus, parahippocampalgyrus, thalamus, and amygdala) in comparison with women without depression [13].

\section{Sexual Dysfunction in Depression}

Depression is characterized by loss of interest, reduction in energy, lowered self-esteem and inability to experience pleasure: irritability and
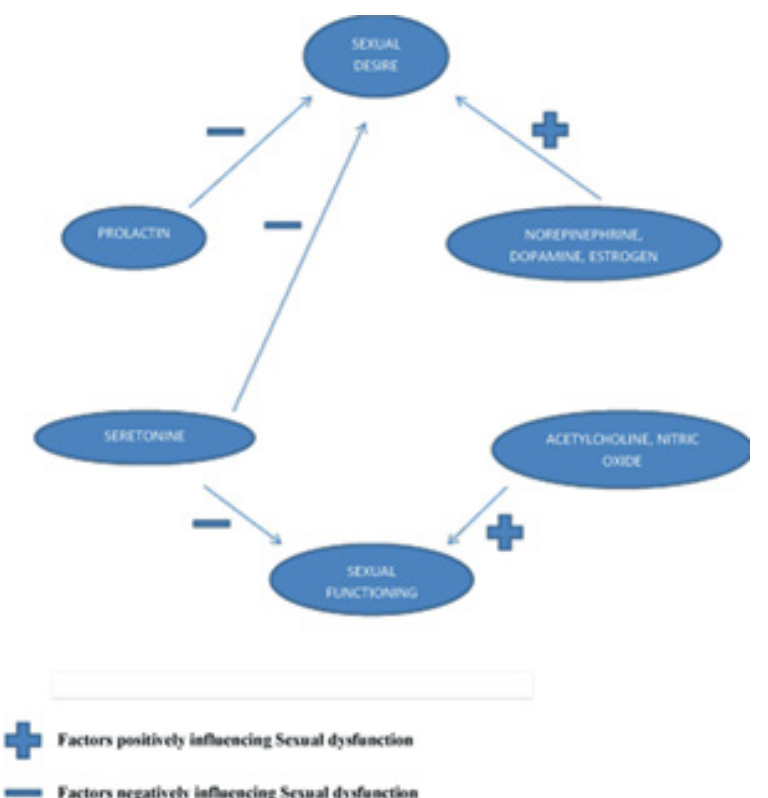

Figure 1: Neurobiological factors influencing sexual response factors. social withdrawal may impair the ability to form and maintain intimate relationships. It would be surprising if this constellation of symptoms did not produce difficulties in sexual relationships [14].

In an early study of 132 patients with depressive disorders, loss of sexual interest (characterized by loss of libido or decrease of sexual desire or potency) was reported by $72 \%$ of patients with unipolar disorder and $77 \%$ of those with bipolar disorder [15]. Loss of sexual desire may be the presenting complaint in some patients who are found to have significant depressive symptoms only after direct questioning. In others, low sexual desire may pre-date other features of depression [16].

Although the incidence of specific types of sexual dysfunction varies across studies, loss of sexual desire may be more common than disorders of arousal and orgasm. For example, in one comparative study, changes in libido were significantly more common in patients with depression, but the prevalence of impotence, orgasmic or ejaculatory problems did not differ from controls [17]. The prospective Zurich cohort study showed that the overall prevalence of sexual problems in subjects with depression (including major depression, dysthymia and recurrent brief depression) was about twice that in controls (50\% vs. 24\%) [18]. This difference encompassed emotional problems, sexual dysfunction and both decreased and increased libido. The study findings were from a group of young people (28-35 years old) and are not necessarily applicable to older age groups.

The Zurich study also compares the prevalence of sexual problems in untreated patients and patients receiving either medication $(50 \%$ benzodiazepines, $50 \%$ antidepressants) or psychotherapy. Sexual problems were more prevalent in the 78 patients who received treatment (62\%) than in the 122 who did not (45\%) and both groups had a higher prevalence of sexual dysfunction than did the 326 controls (26\%). No statistically significant differences were found in the prevalence of any form of sexual dysfunction between patients treated with medication or psychotherapy alone [19].

\section{Pathophysiology of Antidepressant Induced Sexual Dysfunction}

The pathophysiology of antidepressant induced SD remains an ongoing area of research. Certain neurotransmitters and neurologic pathways have been implicated in the cause of antidepressant associated SD. Neurotransmitters like seretonin and dopamine has significant impact on sexual functioning, glutamate, nitric oxide, prolactin, and acetylcholine also seem to play a role [20].

Parasympathetic nervous system with major contribution from lumbar spinal cord is largely involved in male erection and female lubrication. Acetylcholine through parasympathetic activation leads to vasodilation in erectile tissues of the penis and clitoris. Nitric oxide also plays an important role. Norepinephrine inhibits erection by binding to peripheral alpha-1 receptors [21]. There are also reports of involvement of cytochrome enzymes in causing sexual dysfunction. Studies have reported a higher incidence of SD with paroxetine in persons who were cytochrome P450 2D6 poor metabolizers. Other drugs like citalopram, escitalopram, sertraline, and to a lesser extent fluoxetine and fluvoxamine are substrates for the cytochrome P450 $2 \mathrm{C} 19$ isoenzyme and it is thought that polymorphisms encoding for this isoenzyme could also play a role in SD. However there is a lack of definitive evidence validating these findings [22-24].

\section{Antidepressants and Sexual Dysfunction}

The evaluation of sexual dysfunction as an impact of medication 
is difficult, as depressive state itself may cause sexual dysfunction. Depression itself causes loss of libido, negatively affects arousal, orgasm, causes emotional withdrawal from love interest and causes subsequent performance anxiety about having sex. Unless the clinician takes sexual history before starting antidepressants, ideally before the start of the illness, separating sexual dysfunction of the mood disorders from those induced by antidepressants is difficult, if not impossible. The other factors like physical comorbidity (e.g. diabetes mellitus, peripheral vascular disease, ischemic heart disease), pre-existing sexual problems (premature ejaculation, anorgasmia) may confound the findings. Reluctance on the part of patient to volunteer sexual information as well as reluctance on the part of therapist to collect sexual information also acts as a confounding factor.

\section{Tricyclic Antidepressants and Sexual Dysfunction}

An earlier study which was a prospective randomized double blind study which used amoxapine in the dose range of $26-70 \mathrm{mg}$ and amitryptaline in the dose range of $64-107 \mathrm{mg}$, the definition of sexual dysfunction in this study was either loss of libido or impotence. Prevalence of sexual dysfunction in amoxapine group was $26 \%$ while in the amitryptaline group was $31 \%$. Major drawback of this study apart from libido and erection, other components of sexual response cycle was not assessed [25]. Another study which was a Randomised double blind placebo controlled study compared tricyclics with monoamino oxidase inhibitors. In this study imipramine was used in the dose range of 200-300 mg, phenlezine in the dose range of 60-90 mg. Sexual functioning questionnaire was used in this study, imipramine group had $30 \%$ sexual dysfunction while phenlazine group had $40 \%$ sexual dysfunction compare $6 \%$ in the placebo group [25].

\section{Selective Serotonin Reuptake Inhibitors and Sexual Dysfunction}

ADRAC study which compared different a SSRIs in terms of sexual dysfunction based on spontaneous reporting found, paroxetine having the highest sexual function when compared to $21 \%$ in sertraline and $19 \%$ in fluvoxetine [26]. Another prospective nonrandomized open label study which compared Fluoxetine, Paroxetine, Fluvoxamine and Sertraline in terms sexual dysfunction, using the definition of sexual dysfunction as decreased libido, delayed or absent orgasm, delayed or absent ejaculation, impotence. In this study paroxetine had the highest sexual dysfunction with $65 \%$, followed by fluvoxamine- $59 \%$, sertraline $56 \%$ and fluvoxetine 54\% [27]. Another prospective randomized double blind study comparing paroxetine and fluvoxamine found $21 \%$ of men who were on paroxetine had impotence compared to $14 \%$ of men who were on fluvoxamine, $21 \%$ of men had ejaculatory abnormality when compared to $7 \%$ of men on fluvoxamine and decreased libido was reported in $17 \%$ of men who were on paroxetine when compared to $13 \%$ who were on fluvoxamine. The major drawback of this study is that there was on use of any standardized sexual dysfunction scale [28].

\section{Role of Agents with Dual Actions on Serotonin and Norepinephrine in Sexual Dysfunction}

There is some evidence to suggest that noradrenergic effects may mitigate the serotonin influence on sexual function, although results of studies evaluating the serotonin norepinephrine reuptake inhibitors (duloxetine, milnacipran, venlafaxine, and desvenlafaxine) have been inconsistent. Comparative studies of duloxetine and SSRIs have shown that treatment - emergent sexual dysfunction was significantly lower with duloxetine than paroxetine orescitalopram but this difference was not maintained after 12 weeks of treatment, suggesting that duloxetine may only have a short term advantage over SSRIs [29-31].

Desvenlafaxine succinate which is a major active metabolite of venlafaxine, seems to have lower sexual adverse effects in women compared with men, although these studies have relied on spontaneous self - report only [32,33]. Mirtazapine stimulates noradrenergic and serotonergic activity by its agonist effects on postsynaptic 5 - HT1A receptors and concurrent antagonist effects on 5 - HT2 and 5- HT3 receptors. The 5- HT2 blockade mechanism is thought to prevent serotonin- mediated adverse effects on sexual function. On the basis of this it is claimed that in comparison to SSRIs and ven afaxine, mirtazapine is significantly less likely to produce sexual dysfunction [34-36]. Ozmenler et al. [37] also reported that when remitted patients with SSRI- induced sexual dysfunction were switched to mirtazapine, approximately half of them reported no sexual dysfunction at the end of the 8- week treatment. There is also preliminary evidence to suggest that mirtazapine improves duloxetine- induced sexual dysfunction $[36,37]$.

Other studies report that when mirtazapine leads to sexual dysfunction, the intensity is significantly lower [38]. In a large sample study, Clayton et al. evaluated more than 6000 patients who had been prescribed newer antidepressants. The highest prevalence of sexual dysfunction was found among patients taking an SSRI, mirtazapine, or venlafaxine, while the lowest prevalence was among patients taking nefazodone and bupropion [2].

\section{Measuring Sexual Dysfunction}

Recognition of antidepressant- related sexual dysfunction in clinical practice is complicated by a number of factors. In particular, sexual side effects often are not spontaneously reported by patients; hence direct inquiry is often required. In addition, even where sexual dysfunction has been identified in a patient receiving antidepressant medication, it may be difficult to distinguish the sexual dysfunction due to depression per se and sexual dysfunction due to antidepressants. Moreover, it is important to remember that the diagnosis of antidepressant induced sexual dysfunction should not be assigned without full consideration of diagnostic alternatives. The use of recreational substances, the effects of co-administered medications, the presence of a primary sexual disorder, presence of a medical condition affecting sexual function, psychosocial factors such as relationship conflict, sexual effects of the depression, presence of a comorbid psychiatric disorder which could contribute to sexual dysfunction should be considered in the differential Diagnosis $[7,39]$.

The most valuable asset in assessing the antidepressant induced nature of a sexual dysfunction is the patient's own history and description [40]. Sexual dysfunction is most obviously a side effect when it is reported as a new symptom after the initiation of antidepressant. Typically, the patient will recover from depression, with remission of virtually all symptoms and return of normal functioning. However, the patient will report either an absence of return of libido or an actual further suppression of sexual interest. The patient may also describe a recent- onset difficulty in achieving organism [41]. In day to day practice, sexual dysfunction associated with antidepressants can be suspected when the onset of sexual dysfunction follows the commencement of treatment, particularly if it persists beyond resolution of other depressive symptoms.

\section{Impact of Sexual Dysfunction}

Studies have evaluated the impact of sexual dysfunction in the form of relationship conflict, marital disharmony, quality of life and 
compliance with treatment. However, there is sparse data with respect to evaluation of impact of antidepressant induced sexual dysfunction in subjects with depression. Multiple clinical observations in general population suggest that sexual dysfunction is strongly associated with relationship conflict $[29,42-44]$. Cross sectional population survey also reveal that sexual problems among men were associated with anxiety and medical problems, whereas for women sexual dysfunction was associated with marital difficulties. Studies evaluating the marital relationship have shown that marital satisfaction was significantly associated with sexual desire [45]. Other researchers consider marital distress as a causal determinant and as an outcome of low sexual desire; low marital satisfaction can reduce motivation for sexual intimacy and perhaps even promote sexual withholding, and lack of sexual desire over time can lead to frustration and dissatisfaction. This reciprocal cycle is thought to lead some couples into an escalating pattern of sexual dysfunction [46]. It is also seen that sexual dysfunction in male partner can trigger negative emotions in the spouse-typical concerns of the female partner being that she is no longer attractive to her partner, that her partner no longer loves her, leads to a frustration at the lack of tenderness in the relationship, communication problems and lack of understanding on both sides. Impact of sexual dysfunction on quality of life has been assessed in psychiatrically ill subjects and these studies have shown that compared to those without sexual dysfunction, subjects with sexual dysfunction have poor quality of life. In subjects with depression, it is seen that impaired sexual functioning is associated with poor quality of life and social functioning beyond disruption attributable to low mood, reduced drive and impaired ability to experience pleasure. In a multicentric study from Europe it was seen that subjects with antidepressant induced sexual dysfunction had significantly worse quality of life on the mental component but not on the physical component. It was further seen that subjects with antidepressant induced sexual dysfunction more frequently reported negative impact on self-esteem, relationship, mood and overall quality of life [47].

\section{Impact of Sexual Dysfunction on Compliance}

One of the important consequences of sexual dysfunction in people taking antidepressants is poor treatment acceptance and compliance [45]. Studies have shown that sexual dysfunction is the 2nd most frequent side effect rated as "extremely difficult to live with" after weight gain and 3rd most frequently reported side effect as a cause of noncompliance [47]. A study involving subjects with severe mental illnesses showed that $62.5 \%$ of men and $38.5 \%$ of women felt that their psychiatric medications were causing sexual side effects and $41.7 \%$ of men and $15.4 \%$ of women admitted that they had stopped their medications at some point during treatment because of sexual side effects [48].

\section{Treatment of Anti-depressant Induced Sexual Dysfunction}

There are various ways to approach treatment of sexual dysfunction associated with antidepressants. The initial and an important step are to determine sexual functioning of the patient. A detailed history has to be obtained. Various factors contributing like medical, psychological and interpersonal factors have to be considered as contributing factors to sexual dysfunctions. One of the approaches is to consider a drug with limited propensity to cause sexual dysfunction. Bupropion, mirtazapine or nefazodone make better choices when compared to other antidepressants. This method has been studied in small open label studies showing improvement in sexual functioning in patients switched to bupropion and mirtazapine. There is a lack of randomized controlled trials to further establish this approach of treatment.
Similarly, early reports suggest of benefits of the addition of buspirone to antidepressants for resolving sexual dysfunction but the same has not been replicated in larger studies. Another approach is temporarily stopping the offending drug as a technique for reversing sexual problems associated with antidepressants. Although this method is useful in a selected group of patients this drug holiday method has limited value because the risks are likely to be greater than the benefits. The only conclusively effective treatment for sexual dysfunctions associated with antidepressants is the addition of Nitric Oxide Synthetase inhibitors like Sildenafil (25-100 mg) for the erectile dysfunction caused by antidepressants. It has also been shown to improve problems associated with interest, orgasm and overall satisfaction when on antidepressants. There are also reports of no loss of antidepressant benefit with the concurrent use of Sildenafil [48].

\section{Conclusion}

Depression by 2020 will be the second most common cause of morbidity. Sexuality is the ultimate union of mind and body. Depression and sexual dysfunction are the most commonly associated co-morbities. Sexual functioning is one of the key determinants of quality of life. Association of sexual dysfunction with depression as well as antidepressant is more common than thought of lack of knowledge among professionals about sexual medicine leads to persistent failure to acknowledge sexual dysfunction in patients. Patients willingly discuss about sexual problems, if the health care professional broaches the topic. By identifying and treating sexual dysfunctions professionals not only help the patients to enhance their quality of life but also make treatment successful.

\section{References}

1. http://www.who.int/healthinfo/paper54.pdf

2. Clayton AH, Pradko JF, Croft HA, Montano CB, Leadbetter RA, et al. (2002) Prevalence of sexual dysfunction among newer antidepressants. J Clin Psychiatry 63: 357-366.

3. Stahl SM (2008) Antidepressant "Stahl's essentials of psycholpharmacology Cambridge Publication, USA.

4. American Psychiatric Association (2000) Practice guideline for the treatment of patients with major depressive disorder (revision). Am J Psychiatry 157: 1-45.

5. Crismon ML, Trivedi M, Pigott TA, Rush AJ, Hirschfeld RM, et al. (1999) The Texas Medication Algorithm Project: Report of the Texas consensus conference panel on medication treatment of major depressive disorder. J Clin Psychiatry 60: $142-156$

6. Marson L, McKenna KE (1990) The identification of a brain stem site controlling spinal sexual reflexes in male rats. Brain Res 515: 303-308.

7. Angst J (1998) Sexual problems in healthy and depressed persons. Int Clin Psychopharmacol 13: S1-S4.

8. Carmichael MS, Humbert R, Dixen J, Palmisano G, Greenleaf W, et al. (1987) Plasma oxytocin increases in human sexual response. J Endocrinol Metab 64 27-31.

9. Roose SP (1999) Tolerability and patient compliance. J Clin Psychiatry 60: 14 17.

10. Croft H, Settle E, Houser T, Batey SR, Donahue RM, et al. (1999) A placebocontrolled comparison of the antidepressants efficacy and effects on sexual functioning of sustained release bupropion and sertraline ClinTher 21: 643-658.

11. Coleman CC, Cunningham LA, Foster VJ (1999) Sexual dysfunction associated with the treatment of depression; A placebo-controlled comparison of bupropion sustained release \& sertraline treatment. Ann Clin Psychiatry 11: 205-215.

12. Kavoussi RJ, Segraves RT, Hughes AR, Ascher JA, Johnston JA, et al. (1997) Double blind comparison of bupropion sustained release and sertraline in depressed out patients J Clin Psychiatry 58: 532-537.

13. Feiger A, Kiev A, Shrivastava RK (1996) Nefazodone versus sertraline in out patients with major depression, focus on efficacy, tolerability \& effects on sexual function \& satisfaction. Eur Neuropsychopharmacol 6: 48. 
Citation: Manohar SJ, Sathyanarayana Rao TS, Chandra S, Maheshwari S, George M, et al. (2017) Sexual Dysfunctions in Depression. Clin Depress 3: 125.

14. Baldwin DS (2001) Depression and sexual dysfunction. Br Med Bull 57: 81-99.

15. Clayton AH, Kornstein SG, Rosas G, Gucio-Pabia C, Tourian KA, et al. (2009) An integrated analysis of the safety and tolerability of desvenlafaxine compared with placebo in the treatment of major depressive disorder. CNS Spectr 14 183-195.

16. Patricia SE, Schiavi RC (1986) Lifetime psychopathology in individuals with low sexual desire. J Nerv Ment Dis 174: 646-651.

17. Mathew RJ, Weinman ML (1982) Sexual dysfunctions in depression. Arch Sex Behav 11: 323-325.

18. Ernst C, Foldenyi M, Angst J (1993) The Zurich Study: XXI. Sexual dysfunctions and disturbances in young adults. Eur Arch Psychiatry Clin Neurosci 243: 179188.

19. Freeman ME, Kanyicska B, Lerant A, Nagy G (2000) Prolactin: structure, function, andregulation of secretion. Physiol Rev 80: 1523-1631.

20. Clayton AH, El Haddad S, lluonakhamhe JP, Ponce Martinez C, Schuck AE, et al. (2014) Sexual dysfunction associated with major depressive disorder and antidepressant treatment. Expert Opin Drug Saf 13: 1361-1374.

21. Kleinberg DL, Davis JM, de Coster R, Van Baelen B, Brecher M, et al. (1999) Prolactin levels and adverse eventsin patients treated with risperidone. $\mathrm{J}$ Clin Psychopharmacol 19: 57-61.

22. Stevenson JM, Bishop JR (2014) Genetic determinants of selective serotonin reuptake inhibitor related sexual dysfunction. Pharmacogenomics 15: 1791 1806.

23. Zourkova A, Hadasova E (2002) Relationship between CYP 2D6 metabolic status and sexual dysfunction in paroxetine treatment. J Sex Marital Ther 28: 451-461.

24. Zourkova A, Ceskova' E, Hadasova' E (2007) Links among paroxetine-induced sexual dysfunctions, gender, and CYP2D6 activity. J Sex Marital Ther 33: 343355.

25. Balon R, Yeragani VK, Pohl R, Ramesh C (1993) Sexual dysfunction during antidepressant treatment. J Clin Psychiatry 54: 209-212.

26. Jacobsen FM (1992) Fluoxetine-induced sexual dysfunction \& an open trial of yohimbine. J Clin Psychiatry 53: 119-122.

27. Montejo-Gonzalez AL, Llorca G, Izqueirodo JA, Ledesma A, Bousono M, et al. (1997) Fluoxetine, Paroxetine, sertraline \& fluvoxamine in a prospective, multicentre and descriptive clinical study of 344 patients. J Sex Marital Ther 23: $176-194$

28. Lee KUK, Lee YM, Nam JM, Lee HK, Kweon YS, et al. (2010) AntidepressantInduced sexual dysfunction among newer antidepressant in a naturalistic selling. Psychiatry Investing 7: 53-59.

29. Rochschild AJ (2000) Sexual side effects of antidepressants. J Clin Psychiatry 61: $28-36$

30. Monteiro WO, Noshirvani HF, Marks IM, Lelliott PT (1987) Anorgasmia from clomipramine in obsessive - compulsive disorder: A controlled trial. $\mathrm{Br} J$ Psychiatry 151: 107-112.

31. Delgado P, Brannan S, Mallinckrodt C, Tran PV, McNamara RK, et al. (2005) Sexual functioning assessed in 4 double blind placebo and paroxetine controlled trials of duloxetine for major depressive disorder. J Clin Psychiatry 66: 686-692.
32. Clayton A, Kornstein S, Prakash A, Mallinckrodt C, Wohlreich M (2007) Changes in sexual functioning associated with duloxetine, escitalopram, and placebo in the treatment of patients with major depressive disorder. $\mathrm{J}$ Sex Med 4: $917-929$.

33. Liebowitz MR, Manley AL, Padmanabhan SK, Ganguly R, Tummala R, et al. (2008) Efficacy, safety, and tolerability of desvenlafaxine $50 \mathrm{mg} /$ day and 100 $\mathrm{mg} /$ day in outpatients with major depressive disorder. Curr Med Res Opin 24 1877-1890.

34. Septien- Velez L, Pitrosky B, Padmanabhan SK, Germain JM, Tourian $\mathrm{KA}$, et al. (2007) A randomized, double - blind, placebo - controlled trial of desvenlafaxine succinate in the treatment of major depressive disorder. Int Clin Psychopharmacol 22: 338-347.

35. Montejo AL, Llorca G, Izquierdo JA, Rico- Villademoros F (2001) Incidence of sexual dysfunction associated with antidepressant agents: a prospective multicenter study of 1022 outpatients. Spanish Working Group for the Study of Psychotropic-Related Sexual Dysfunction. J Clin Psychiatry 62: 10-21.

36. Werneke U, Northey S, Bhugra D (2006) Antidepressants and sexual dysfunction. Acta Psychiatr Scand 114: 384-397.

37. Ozmenler NK, Karlidere T, Bozkurt A, Yetkin S, Doruk A, et al. (2008) Mirtazapine augmentation in depressed patients with sexual dysfunction due to selective serotonin reuptake inhibitors. Hum Psychopharmacol 23: 321-326.

38. Ravindran LN, Eisfeld BS, Kennedy SH (2008) Combining mirtazapine and duloxetine in treatment - resistant depression improves outcomes and sexual function. J Clin Psychopharmacol 28: 107-108.

39. Kennedy S, Dickens S, Eisfeld B, Bagby RM (1999) Sexual dysfunction before antidepressant therapy in major depression. J Affect Disord 56: 201-208.

40. Gelenberg AJ, Delgado P, Nurnberg HG (2000) Sexual side effects of antidepressant drugs. Curr Psychiatr Rep 2: 223-227.

41. Segraves RT (1989) Effects of psychotropic drugs on human erection and ejaculation. Arch Gen Psychiatry 46: 275-284.

42. Hartman LM (1980) The interface between sexual dysfunction and marital conflict. Am J Psychiatry 137: 576-579.

43. Hartman LM (1980) Relationship factors and sexual dysfunction: Implications for assessment and treatment. Can J Psychiatry 25: 560-563.

44. McCarthy BW (1998) Sex in the first two years of marriage. J Fam Psychother 9: 1-11.

45. Ashton AK, Jamerson BD, Weinstein WL, Wagoner C (2005) Antidepressantrelated adverse effects impacting treatment compliance: Results of a patient survey. Curr Ther Res Clin Exp 66: 96-106.

46. Williams VS, Baldwin DS, Hogue SL, Fehnel SE, Hollis KA, et al. (2006) Estimating the prevalence and impact of antidepressant- induced sexua dysfunction in 2 European countries: A cross - sectional patient survey. J Clin Psychiatry 67: 204-210.

47. Rosenberg KP, Bleiberg KL, Koscis J, Gross C (2003) A Survey of sexual side effects among severely mentally ill patients taking psychotropic medications: Impact on compliance. J Sex Marital Ther 29: 289-296.

48. Balon R (2008) Sexual dysfunction. The brain-body connection. Adv Psychosom Med Basel, Karger 29: 107-130. 